\title{
Influência do turno laboral na formação de edema dos membros inferiores em indivíduos normais
}

\author{
Influence of working shift on the formation of lower limb edema in \\ normal individuals
}

\author{
Cleusa Ema Quilici Belczak ${ }^{1}$, José Maria Pereira de Godoy ${ }^{2}$, Rubiana Neves Ramos ${ }^{3}$, \\ Márcia Aparecida de Oliveira ${ }^{3}$, Sergio Quilici Belczak ${ }^{4}$, Roberto Augusto Caffaro ${ }^{5}$
}

\section{Resumo}

Contexto: A presença de edema vespertino nos membros inferiores de indivíduos normais, após jornada habitual de trabalho, foi demonstrada na literatura nacional e internacional. O ritmo de formação e o acúmulo desse edema variam de acordo com os distintos turnos laborais.

Objetivo: $\mathrm{O}$ edema de membros inferiores tem sido descrito após jornadas habituais de trabalho e representa uma queixa freqüente na prática vascular. O objetivo deste estudo foi avaliar a evolução do edema em indivíduos normais durante os distintos turnos laborais.

Método: Foram feitas avaliações volumétricas de ambos os membros inferiores em 20 profissionais da área da saúde do Hospital e Maternidade São Marcos de Maringá, no Paraná. A escolha dos participantes foi por ordem de chegada, e as volumetrias foram feitas por técnica de deslocamento de água às 7,13 e $19 \mathrm{~h}$. Para análise estatística foi utilizado o teste $t$ de Student, considerando erro alfa de $5 \%$.

Resultado: Dos 20 participantes, 19 eram do sexo feminino e 1 do masculino, sem evidência de doença venosa nos membros inferiores e pertencentes a $\mathrm{C} 0$ e $\mathrm{C} 1$ da classificação $\mathrm{CEAP}(\mathrm{C}=$ clínica, $\mathrm{E}=$ etiologia, $\mathrm{A}=$ segmento anatômico, $\mathrm{P}=$ fisiopatologia). As idades dos participantes variaram entre 20 e 53 anos. Detectou-se aumento significativo de volume nos membros inferiores entre os distintos períodos avaliados, com $\mathrm{p}=0,0001$ e 0,0001 , respectivamente. A maior variação ocorreu no período da manhã, com média \pm desvio padrão de $107,2 \pm 63,5 \mathrm{~mL}$, enquanto que à tarde, a variação foi de $44,5 \pm 35,4 \mathrm{~mL}$

Conclusão: O edema é uma constante durante atividades laborais, mesmo em pessoas sem doença venosa manifesta e sofre influência do turno laboral ao qual o trabalhador se encontra exposto.

Palavras-chave: Edema, insuficiência venosa, fisiologia do trabalho, turno laboral.

\section{Abstract}

Background: Presence of evening edema of lower limbs in normal individuals after a regular working shift has been shown in the national and international literature. Formation rhythm and accumulation of this type of edema vary according to different work shifts.

Objective: Edema of the legs has been described after regular work shifts and is a common complaint in vascular practice. The objective of this study was to evaluate the evolution of edema in apparently normal individuals during distinct work shifts.

Method: Volumetric assessments of both legs were performed in 20 healthcare professionals at São Marcos Hospital and Maternidade in Maringá, Brazil. Individuals were selected on a consecutive basis with water displacement volumetry being performed at 7:00 a.m., 1:00 p.m. and 7:00 p.m. Student's t test was used for statistical analysis with an alpha error of $5 \%$ being considered acceptable.

Results: The study group consisted of 19 women and one man with ages ranging from 20 to 53 years old. The participants had no evidence of venous diseases of the lower limbs and were $\mathrm{C} 0$ or $\mathrm{C} 1$ according to the CEAP classification $(\mathrm{C}=$ clinical, $\mathrm{E}=$ etiology, $\mathrm{A}=$ anatomy, $\mathrm{P}=$ pathophysiology). Significant increases in the volume of both legs were seen after both shifts ( $p=0.0001$ in both cases). The greatest variation occurred during the morning with a mean \pm standard deviation of $107.2 \pm 63.5 \mathrm{~mL}$, while in the afternoon mean increase was $44.5 \pm 35.4 \mathrm{~mL}$.

Conclusion: Edema is constant in work activities, even in individuals without obvious venous diseases, and is influenced by work shift. Khift.

Keywords: Edema, venous insufficiency, physiology of work, work

1. Doutoranda em Cirurgia Geral, Faculdade de Ciências Médicas da Santa Casa de São Paulo (FCMSCSP), São Paulo, SP. Professora, Pós-Graduação em Reabilitação Linfovenosa, Faculdade de Medicina de São José do Rio Preto (FAMERP), São José do Rio Preto, SP.

2. Doutor em Cardiologia e Cirurgia Cardiovascular. Livre-docente, professor adjunto, Departamento de Cirurgia, FAMERP, São José do Rio Preto, SP. Pesquisador, Conselho Nacional de Desenvolvimento Científico e Tecnológico (CNPq).

3. Fisioterapeutas, Centro Vascular João Belczak, Maringá, PR.

4. Residente em Cirurgia Vascular, Hospital das Clínicas da Universidade de São Paulo (HCUSP), São Paulo, SP.

5. Doutor em Cirurgia Vascular. Professor adjunto, FCMSCSP, São Paulo, SP.

Não foram declarados conflitos de interesse associados à publicação deste artigo.

Artigo submetido em 03.05.08, aceito em 24.06.08.

J Vasc Bras. 2008;7(3):225-230.

Copyright@ 2008 by Sociedade Brasileira de Angiologia e de Cirurgia Vascular 


\section{Introdução}

Em indivíduos normais, a presença de edema nos membros inferiores (MMII) após jornada habitual de trabalho tem sido avaliada e constatada em alguns estu$\operatorname{dos}^{1,2}$. A formação de edema e sua prevenção com uso de meias elásticas também já foi demonstrada ${ }^{2}$. Outros pesquisadores detectaram variações volumétricas nos MMII ao longo do dia trabalhado, em pacientes hígidos, cujos números diferem entre os membros dominantes e não dominantes e sofrem influência pelo uso de medicações, como por exemplo, os bloqueadores de canal de cálcio ${ }^{3-5}$.

Sabe-se que existe variação hemodinâmica venosa ao longo do dia como conseqüência do afastamento das cúspides valvares, o que promove um aumento dos refluxos venosos ${ }^{6}$. O ritmo circadiano é conhecido como parte da fisiologia humana em muitos sistemas, sejam esses influenciados ou não pela postura ou por variações da bioquímica sangüínea, como por exemplo, no caso dos níveis estrogênicos atuando sobre as células musculares lisas ${ }^{7-9}$.

Considera-se o edema de MMII como importante fator de queda na qualidade de vida, pois gera grande desconforto, cansaço precoce e sensação de peso, o que certamente diminui o rendimento profissional ${ }^{10}$. Tais manifestações clínicas quase sempre representam uma primeira manifestação de descompensação do sistema venoso, cuja complicação grave culmina com o surgimento de insuficiência venosa crônica (IVC) dos MMII, enfermidade a ser prevenida, pois representa, no Brasil, a $14^{\mathrm{a}}$ causa de absenteísmo laboral, razão de constrangimento e de prejuízo econômico tanto para o indivíduo como para a coletividade ${ }^{11}$.

Justifica-se, deste modo, não só a pesquisa das características etiopatogênicas do edema como também que se proceda à quantificação de sua magnitude, para embasar a pesquisa de métodos profiláticos eficazes que evitem a evolução mórbida da IVC e para que se interprete sua expressão clínica quanto ao período de trabalho ao qual o indivíduo está exposto.

O objetivo deste estudo é detectar em indivíduos normais a presença de edema, acumulado ao longo da jornada de trabalho, e avaliar a evolução do mesmo durante os distintos turnos laborais.

\section{Método}

De 10 de dezembro de 2007 a 20 de janeiro de 2008, foram feitas avaliações volumétricas dos MMII em 20 profissionais da área da saúde do Hospital e Maternidade São Marcos de Maringá, no Paraná. Foram avaliados 19 mulheres e 1 homem, com idades que variavam entre 20 e 53 anos, incluindo-se enfermeiros, zeladoras, recepcionistas, copeiras e circulantes de centro cirúrgico. As atividades laborais dos participantes intercalavam períodos de ortostatismo estático com dinâmico e posição sentada, em semelhante proporção de tempo. A seleção foi realizada por convites, de forma consecutiva a partir da chegada ao hospital, excluindo-se aqueles funcionários que permanecessem em bipedestação prolongada ou sentados em todo o período de trabalho. Os participantes foram recrutados no dia de seu plantão de 12 horas. Avaliou-se cada indivíduo antes do início do turno do trabalho, às $7 \mathrm{~h}$, após o turno da manhã, às $13 \mathrm{~h}$, e no final do turno, às $19 \mathrm{~h}$, depois de 12 horas de trabalho, perfazendo um total de 120 medidas em 40 membros estudados.

A volumetria foi feita utilizando-se a técnica de deslocamento de água pelos MMII (pés e pernas). No estudo em questão, todos os participantes faziam parte do mesmo grupo populacional urbano e assinaram um termo de consentimento após terem sido informados sobre o objetivo deste estudo, que foi previamente submetido à apreciação e posterior aprovação do Comitê de Ética da do Centro Universitário de Maringá (PR).

Para evitar a inclusão de pessoas portadoras de IVC dos MMII na amostra, (fator que poderia interferir na quantidade do volume de edema acumulado nos MMII), somente foram admitidos participantes com membros classificados nas categorias $\mathrm{C} 0$ e $\mathrm{C} 1$ da graduação CEAP do Consenso Protocolado do Comitê Internacional em Doença Venosa (no qual $\mathrm{C}=$ clínica, $\mathrm{E}=$ etiologia, $\mathrm{A}=$ segmento anatômico e $\mathrm{P}=$ fisiopatologia), onde $\mathrm{C} 0$ equivale à ausência de sinais e sintomas de insuficiência venosa e $\mathrm{C} 1$ à presença somente de telangiectasias ${ }^{12-14}$. A anamnese e o exame clínico dos indivíduos selecionados foram realizados por médica vascular do hospital.

Excluíram-se também participantes isquêmicos, hipertensos, diabéticos e/ou aqueles que estavam sob uso de medicamentos, como diuréticos e hormônios, que 


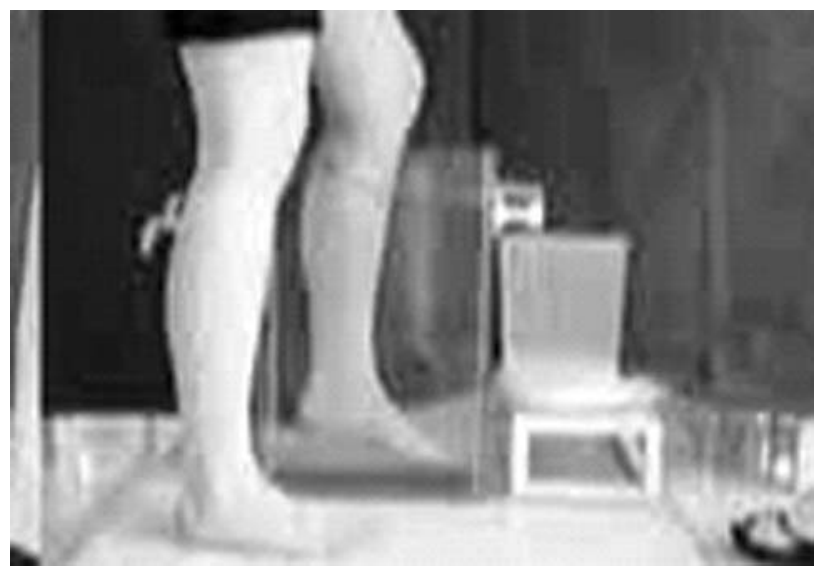

Figura 1 - Cuba de vidro com duas saídas, especialmente construída para realização de volumetria por deslocamento de água

também poderiam influenciar na formação de edema, além dos portadores de edema dos MMII de origem sistêmica, como insuficiência cardíaca congestiva, renal, hepática, mixedema e doenças articulares traumáticas ou reumáticas. Também foram excluídos os indivíduos que tivessem linfedema ou que fizessem uso rotineiro de meias elásticas ou ainda que apresentassem qualquer tipo de lesão dermatológica.

As medidas volumétricas foram realizadas sempre pela mesma fisioterapeuta, utilizando cubas de vidro com água em temperatura ambiente, construídas para tal finalidade. As cubas possuem duas saídas: uma para o nivelamento inicial da água e outra para a saída da água deslocada, sendo previamente avaliado o volume líquido equivalente a essa distância entre as duas saídas, permitindo a perfeita estabilização do nível líquido antes e durante o exame. Considera-se que o volume do membro imerso seja equivalente ao volume de água deslocada para cima na cuba mais o volume de água que sai para o recipiente graduado, onde se medem em números absolutos os mililitros excedentes (Figura 1) ${ }^{1}$.

\section{Resultados}

A Tabela 1 mostra as variações volumétricas dos membros no transcorrer do dia.

A Tabela 2 mostra as médias, desvio padrão, valores mínimos e máximos, mediana e $p$ do teste $t$ de Student.

\section{Discussão}

O presente estudo demonstra as variações volumétricas dos MMII em indivíduos sem doença venosa aparente, nos quais o edema é uma constante no transcorrer do dia, sendo mais significativo no primeiro período de turno de trabalho, ou seja, pela manhã, conforme pode-se observar na Tabela 2. Para medir a formação de edema, optou-se pela volumetria por deslocamento de água, por ser método seguro, de baixo custo, passível de repetição, facilmente executável e considerado, ainda hoje, como o gold standard para esse tipo de aferição ${ }^{15-18}$.

Apesar de o edema estar associado a muitas enfermidades sistêmicas e a causas locais que em nossa amostra foram fatores de exclusão, nosso estudo alerta para o fato de ele estar presente em trabalhadores aparentemente saudáveis. Na literatura pesquisada, foi demonstrado que o edema se deve também à postura adotada durante a jornada de trabalho ${ }^{14}$. Sabe-se que as alterações volumétricas durante toda a atividade diária têm como principal agravante o ortostatismo prolongado. Assim, incluímos em nossa pesquisa apenas indivíduos de atitude laboral semelhante, conforme descrito anteriormente, e excluímos aqueles que se mantinham estaticamente por longos períodos em uma mesma posição, como cirurgiões, instrumentadores e secretárias executivas, pela possibilidade de interferência nos resultados obtidos.

Independentemente de sua origem ou etiopatogenia, é importante que na prática clínica a quantidade de edema nos MMII seja avaliada antes e após o indivíduo executar sua jornada de trabalho, e que se investigue se o turno no qual o indivíduo exerce sua função interfere na quantidade de edema formado. É comum encontrar indivíduos com sintomas sugestivos de IVC sem doença venolinfática estabelecida. Acredita-se que o ritmo de variação volumétrica do membro, no transcorrer do dia, poderia justificar tais sintomas. Nesses casos, a volumetria poderia ser sugerida e, sendo constatadas alterações, medidas profiláticas poderiam ser prescritas (por exemplo, intervalo com repouso em decúbito, no meio do período laboral, para baixar a pressão venosa e/ou uso adequado de meias elásticas).

Nossas avaliações foram efetuadas em um único período sazonal; sabe-se que o edema é sensível não só às 
Tabela 1 - Volumetria dos membros inferiores, em mililitros, nos distintos turnos laborais, realizada às 7 , 13 e às 19 h, respectivamente, durante jornada de trabalho dupla de 12 horas

\begin{tabular}{|c|c|c|c|c|c|}
\hline \multicolumn{2}{|c|}{ Entrada (7 h) } & \multicolumn{2}{|c|}{ Metade do turno (13 h) } & \multicolumn{2}{|c|}{ Final do turno (19 h) } \\
\hline MID & MIE & MID & MIE & MID & MIE \\
\hline 2775 & 2805 & 2915 & 2895 & 2995 & 3005 \\
\hline 3075 & 3025 & 3215 & 3175 & 3225 & 3225 \\
\hline 2725 & 2655 & 2875 & 2825 & 2925 & 2865 \\
\hline 2925 & 2925 & 2985 & 2945 & 3075 & 2985 \\
\hline 3675 & 3625 & 3825 & 3825 & 3875 & 3925 \\
\hline 2425 & 2525 & 2625 & 2625 & 2755 & 2705 \\
\hline 2385 & 2465 & 2475 & 2545 & 2555 & 2575 \\
\hline 1995 & 2005 & 2115 & 2055 & 2135 & 2075 \\
\hline 2515 & 2545 & 2555 & 2655 & 2575 & 2585 \\
\hline 3275 & 3365 & 3445 & 3515 & 3535 & 3565 \\
\hline 2775 & 2825 & 2845 & 2885 & 2875 & 2915 \\
\hline 2405 & 2455 & 2525 & 2665 & 2605 & 2695 \\
\hline 3165 & 3255 & 3205 & 3275 & 3205 & 3285 \\
\hline 2345 & 2265 & 2425 & 2375 & 2455 & 2415 \\
\hline 2665 & 2685 & 2745 & 2725 & 2775 & 2765 \\
\hline 3375 & 3405 & 3665 & 3605 & 3705 & 3655 \\
\hline 3175 & 3375 & 3235 & 3405 & 3255 & 3425 \\
\hline 2975 & 2805 & 3145 & 2925 & 3225 & 2975 \\
\hline 3335 & 3335 & 3365 & 3345 & 3375 & 3365 \\
\hline 2875 & 2775 & 2945 & 2875 & 2995 & 2925 \\
\hline
\end{tabular}

MID = membro inferior direito; MIE = membro inferior esquerdo.

Tabela 2 - Valores de média, desvio padrão, mínimo e máximo e mediana das variáveis mensuradas, segundo o grupo de estudo

\begin{tabular}{|c|c|c|c|c|c|c|c|}
\hline Variável/grupo & $\mathbf{n}$ & Média & DP & Mínimo & Máximo & Mediana & p \\
\hline \multicolumn{8}{|c|}{ Volume de entrada } \\
\hline Manhã & 40 & 2849,5 & 421,3 & 1995,0 & 3675,0 & 2805,0 & \\
\hline Tarde & 40 & 2956,8 & 433,57 & 2055,0 & 3825,0 & 2905,0 & \\
\hline Noite & 40 & 3001,8 & 438,0 & 2075,0 & 3925,0 & 2980,0 & \\
\hline \multicolumn{8}{|l|}{ Diferenças } \\
\hline Tarde (13 h) & 40 & $-107,2$ & 63,5 & $-290,0$ & $-10,0$ & $-127,5$ & $0,0001 *$ \\
\hline Noite (19 h) & 40 & $-44,5$ & 35,4 & $-130,0$ & 70,0 & $-40,0$ & $0,0001^{*}$ \\
\hline
\end{tabular}

$\mathrm{DP}=$ desvio padrão.

* Nível descritivo de probabilidade do teste $t$ de Student. 
alterações da postura, mas também à temperatura ${ }^{16}$. Vayssairat et al. ${ }^{19}$, em 1994, medindo por volumetria voluntários normais, como em nosso estudo, e pacientes com varizes, observaram que também nas pernas normais ocorria diferença significativa entre as medidas da manhã e da tarde. Este fato pode corroborar a modificação da hemodinâmica venosa detectada por vários autores em membros normais durante a atividade de trabalho cotidiana, sendo que existe uma plena concordância com relação à influência do ortostatismo prolongado, da deambulação reduzida ou da posição sentada por períodos extensos ${ }^{6,9,20}$.

Outro aspecto importante a ser considerado é a permeabilidade capilar, pois ela pode promover grandes alterações no volume dos membros sujeitos ao sedentarismo. Diferentemente do volume plasmático, cujo controle se faz a partir de barorreceptores no território arterial e receptores de volume nos territórios de baixa pressão, o controle do fluido intersticial dá-se por meio de forças físicas locais ${ }^{21}$. É preciso que se considerem os níveis de filtração e reabsorção dos fluidos nos tecidos, cujo desequilíbrio constitui a gênese das patologias venolinfáticas. Importa que, em se tratando de estudar o fator edema, sempre se recorde do balanço entre pressões hidrostáticas e coloidosmóticas do interstício e do plasma descrito por Starling em 1896 (apud Szuba et al. ${ }^{22}$ ).

Durante o sono, ou seja, na posição de decúbito mantida por horas no descanso noturno, a redução da pressão gravitacional permite que o membro se esvazie, isto é, que volte ao normal e estabilize as pressões em nível microcirculatório, equilibrando o intra com o extravascular. Supõe-se que pela manhã, uma vez reabsorvidos os edemas do dia anterior, provavelmente a pressão intersticial estará bastante baixa e facilitará a filtração capilar, o que explica a maior formação de edema no turno matutino constatada em nosso estudo, com média de acúmulo de edema de 107,2 mL nesse período. Durante a tarde, graças ao aumento da pressão intersticial resultante dessa elevada filtração da manhã, ocorreria uma resposta compensatória, promovendo uma maior reabsorção para o intravascular, o que justifica, em níveis pressóricos, a menor formação de edema no segundo turno, fato também observado em nossa amostra, cujo acúmulo médio de edema nesse período foi de 44,5 mL. Assim, pode-se imaginar que, de manhã cedo, o membro, ao estar desinchado, comporta-se como uma esponja vazia, e que, ao meio-dia, quando ele já se encontra parcialmente preenchido, não permitirá a formação de uma mesma quantidade de edema, explicando os valores significativos encontrados.

Pode-se afirmar que o volume de edema acumulado na manhã é maior que o da tarde, devido a uma alteração no balanço de pressões por limitação da filtração capilar no segundo período, produzindo uma lentificação da drenagem linfovenosa.

\section{Conclusão}

Conclui-se que o edema é uma constante durante atividades laborais mesmo em pessoas sem doença venosa manifesta, e ele sofre influência do turno laboral ao qual o trabalhador se encontra exposto.

\section{Referências}

1. Belczak CEQ, Godoy JMP, Seidel AC, Silva JA, Cavalheri G Jr., Belczak SQ. Influência da atividade diária na volumetria dos membros inferiores medida por perimetria e por pletismografia de água. J Vasc Bras. 2004;3:304-10.

2. Partsch H, Winiger J, Lun B. Compression stockings reduce occupational leg swelling. Dermatol Surg. 2004;30:737-43.

3. Nilsson S, Haugen GB. Volumetry in the evaluation of swelling in the ankle and the foot. J Oslo City Hosp. 1981;31:11-5.

4. Goldie IF, Gunterberg B, Jacobsen C. Foot volumetry as an objective test of the effect of antiphlogistic drugs in ankle sprains. A preliminary study. Rheumatol Rehabil. 1974;13:204-7.

5. van Hamersvelt HW, Kloke HJ, de Jong DJ, Koene RA, Huysmans FT. Oedema formation with the vasodilators nifedipine and diazoxide: direct local effects or sodium retention? J Hypertens. 1996;14:1041-5.

6. Katz ML, Comerota AJ, Kerr RP, Caputo GC. Variability of venous hemodynamics with daily activity. J Vasc Surg. 1994;19:361-5.

7. Muller JE, Tofler GH, Stone PH. Circadian variation and triggers of onset of acute cardiovascular disease. Circulation. 1989;79:733-43.

8. Tsementzis SA, Gill JS, Hitchock ER, Gill SK, Beevers DG. Diurnal variation of and activity during the onset of stroke. Neurosurgery. 1985;17:901-4.

9. Brijker F, Heijdra YF, Van Den Elshout FJ, Bosch FH, Folgering HT. Volumetric measurements of peripheral oedema in clinical conditions. Clin Physiol. 2000;20:56-61. 
10. Belczak CEQ, Cavalheri G Jr., Godoy JMP, Caffaro RA, Belczak SQ. Relação entre a mobilidade da articulação talocrural e a úlcera venosa. J Vasc Bras. 2007;6:149-55.

11. Castro Mde C. Chronic venous insufficiency of the lower limbs and its socio-economic significance. Int Angiol. 1991;10:152-7.

12. Beebe HG, Bergan JJ, Bergqvist D, et al. Classification and grading of chronic venous disease in the lower limbs. A consensus statement. Int Angiol. 1995;14:197-201.

13. Partsch H. Classificação da insuficiência venosa crônica. CEAP. In: Thomaz JB, Belczak CE, editores. Tratado de flebologia e linfologia. Rio de Janeiro: Rubio; 2006. p. 85-9.

14. França LHG, Tavaraes V. Insuficiência venosa crônica. Uma atualização. J Vasc Bras. 2003;2:318-28.

15. Stranden E. A comparison between surface measurements and water displacement volumetry for the quantification of leg edema. J Oslo City Hosp. 1981;31:153-5.

16. Oliveira AB, Lara CP, Lins SS, Cunha Filho IT. Comparação entre as medidas inferenciais de edema de membros inferiores utilizando o leg-o-meter e o deslocamento de água. Rev Bras Fisioter. 2006;10:43-9.
17. Perrin M, Guex JJ. Edema and leg volume: methods of assessment. Angiology. 2000;51:9-12.

18. Botta G, Arpaia G, Monache GD. La pletismografia. In: Mancini S, editor. Trattato di flebologia e linfologia. Torino: Utet; 2001. p. 273-8.

19. Vayssairat M, Maurel A, Gouny P, Baudot N, Gaitz JP, Naussaume O. La volumetrie: une méthode precise de quantification en phlébologie. J Mal Vasc. 1994;19:108-10.

20. Eifell RK, Ashour HY, Heslop PS, Walker DJ, Lees TA. Association of 24 hour activity levels with the clinical severity of chronic venous desease. J Vasc Surg. 2006;44(3):580-7.

21. Aukland K. Is extracellular fluid volume regulated? Acta Physiol Scand Suppl. 1989;583:59-67.

22. Szuba A, Shin WS, Strauss HW, Rockson S. The third circulation: radionuclide lymphoscintigraphy in the evaluation of lymphedema. J Nucl Med. 2003;44:43-57.

Correspondência:

Cleusa Ema Quilici Belczak

Av. Tiradentes, 1081

CEP 87013-260 - Maringá, PR

E-mail: belczak@wnet.com.br 